\title{
A healthy eating score is inversely associated with depression in older adults: results from the Chilean National Health Survey 2016-2017
}

\author{
Fabian Lanuza ${ }^{1,2,3} \odot$, Fanny Petermann-Rocha ${ }^{4,5} \oplus_{(1)}$ Carlos Celis-Morales ${ }^{4,6,7}{ }_{\odot}$, \\ Yeny Concha-Cisternas ${ }^{8,9}$, Gabriela Nazar ${ }^{10,11}$, Claudia Troncoso-Pantoja ${ }^{12}$, \\ Nicole Lassere-Laso ${ }^{13}$, María Adela Martínez-Sanguinetti ${ }^{14}$, Solange Parra-Soto ${ }^{4}$, \\ Raul Zamora-Ros ${ }^{1,15} \odot$, Cristina Andrés-Lacueva ${ }^{1,2} \oplus$ and Tomás Meroño ${ }^{1,2, *} \oplus$
}

'Biomarkers and Nutrimetabolomics Laboratory, Department of Nutrition, Food Sciences and Gastronomy, Food Technology Reference Net (XIA), Nutrition and Food Safety Research Institute (INSA), Faculty of Pharmacy and Food Sciences, University of Barcelona, Avinguda Joan XXIII, 27-31, Barcelona, 08028, Spain: ${ }^{2}$ Centro de Investigación Biomédica en Red de Fragilidad y Envejecimiento Saludable (CIBERFES), Instituto de Salud Carlos III, Spain: ${ }^{3}$ Centro de Epidemiología Cardiovascular y Nutricional (EPICYN), Facultad de Medicina, Universidad de La Frontera, Temuco, Chile: ${ }^{4}$ Institute of Health and Wellbeing, University of Glasgow, Glasgow, UK: ${ }^{5}$ Facultad de Medicina, Universidad Diego Portales, Santiago, Chile: ${ }^{6}$ Center for Exercise Physiology Research (CIFE), University Mayor, Santiago, Chile: ${ }^{7}$ Laboratorio de Rendimiento Humano, Grupo de Estudio en Educación, Actividad Física y Salud (GEEAFyS), Universidad Católica del Maule, Talca, Chile: ${ }^{8}$ Escuela de Kinesiología, Facultad de Salud, Universidad Santo Tomás, Chile: ${ }^{9}$ Pedagogía en Educación Física, Facultad de Educación, Universidad Autónoma de Chile, Chile: ${ }^{10}$ Departamento de Psicología, Universidad de Concepción, Concepción, Chile: ${ }^{11}$ Centro de Vida Saludable, Universidad de Concepción, Concepción, Chile: ${ }^{12}$ Centro de Investigación en Educación y Desarrollo (CIEDE-UCSC), Departamento de Salud Pública, Facultad de Medicina, Universidad Católica de la Santísima Concepción, Concepción, Chile: ${ }^{13}$ Escuela de Nutrición y Dietética, Facultad de Salud, Universidad Santo Tomás, Chile: ${ }^{14}$ Instituto de Farmacia, Facultad de Ciencias, Universidad Austral de Chile, Valdivia, Chile: ${ }^{15}$ Unit of Nutrition and Cancer, Cancer Epidemiology Research Program, Catalan Institute of Oncology (ICO), Bellvitge Biomedical Research Institute (IDIBELL), Barcelona, Spain

Submitted 3 August 2021: Final revision received 1 December 2021: Accepted 9 December 2021: First published online 13 December 2021

\begin{abstract}
Objective: To investigate the relationship of a healthy eating score with depression in Chilean older adults.

Design: Cross-sectional study.

Setting: Older adults from the Chilean National Health Survey 2016-2017. Associations were analysed using complex samples multivariable logistic regressions adjusted for age, sex, socio-demographic, lifestyles (physical activity, smoking, alcohol consumption and sleep duration), BMI and clinical conditions (hypertension, diabetes, hypercholesterolaemia and cardiovascular diseases). Participants: The number of participants was 2031 ( $\geq 60$ years). The Composite International Diagnostic Interview-Short Form was applied to establish the diagnosis of major depressive episode. Six healthy eating habits were considered to produce the healthy eating score (range: 0-12): consumption of seafood, whole grain, dairy, fruits, vegetables and legumes. Participants were categorised according to their final scores as healthy $(\geq 9)$, average (5-8) and unhealthy $(\leq 4)$.

Results: Participants with a healthy score had a higher educational level, physical activity and regular sleep hours than participants with an average and unhealthiest healthy eating score. Participants classified in the healthiest healthy eating score had an inverse association with depression (OR: 0.28, (95\% CI 0.10, 0.74)). Food items that contributed the most to this association were legumes (15.2\%) and seafood (12.7\%). Conclusion: Older adults classified in the healthiest healthy eating score, characterised by a high consumption of legumes and seafood, showed a lower risk for depression in a representative sample of Chilean population.
\end{abstract}

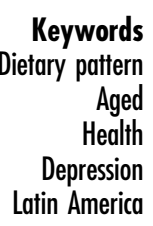


In the next three decades, the proportion of the world's older adults is estimated to double, from $11 \%$ to $22 \%$ of the population by $2050^{(1)}$. Currently, over $20 \%$ of adults aged 60 or over suffer from a mental health disorder ${ }^{(2)}$, and this prevalence is also expected to increase globally. Within mental health disorders, depression is among the top ten leading causes of disability worldwide ${ }^{(3,4)}$. Moreover, depression is associated with comorbidity in many chronic diseases, and it is additionally linked to higher health care costs, disability and mortality ${ }^{(5)}$.

In Chile, the prevalence of a major depressive episode (MDE), diagnosed by the Composite International Diagnostic Interview-Short Form (CIDI-SF), has increased from $11 \cdot 1$ to $16 \cdot 8 \%$ between 2003 and $2016^{(6,7)}$. This proportion is significantly higher than in other countries, and argues for the need of new comprehensive strategies to reduce its incidence ${ }^{(8,9)}$. In fact, in a cross-national epidemiology study carried out in eighteen countries of the World Mental Health Survey Initiative, the mean prevalence of MDE, measured using the CIDI, varied from $5.5 \%$ to $5.9 \%$ in high-income and low-middle income countries, respectively ${ }^{(8)}$. Similarly, a study including twenty-seven European countries reported a prevalence of depressive disorder of $6.4 \%$, using the eight-item Patient Health Questionnaire depression scale ${ }^{(9)}$.

Several behavioural factors have been correlated to MDE. Among them, a healthy diet like the Mediterranean diet has been associated with a lower risk for several mental health diseases ${ }^{(10,11)}$. A total of twenty longitudinal and twenty-one cross-sectional studies were included in a meta-analysis that has reported that healthy dietary indexes were associated with lower risk for depression ${ }^{(10)}$. Moreover, a recent systematic review has demonstrated that adherence to a Mediterranean diet is associated with improvement in mental health ${ }^{(11)}$. Thus, a healthy dietary pattern could contribute to reducing the risk for depression, and supporting medical treatment of depression, partially decreasing undesirable side effects ${ }^{(12)}$. Nonetheless, studies linking dietary patterns and depression risk are heterogeneous, and findings remain inconclusive ${ }^{(13)}$ with no studies in some regions such as Latin America ${ }^{(14)}$.

A recent study from Chile that examined the trajectories of unhealthy foods and alcohol consumption during COVID-19 lockdown observed that younger women and depression symptoms were associated with increases over time in unhealthy food consumption ${ }^{(15)}$. Hence, to our knowledge, this is the first study to investigate the association between healthy foods and depression risk in Chilean older adults. Moreover, the current focus of nutritional epidemiological studies is exploring dietary patterns or food groups instead of specific foods because this reductionist approach may not consider synergistic or antagonistic effects from the whole diet ${ }^{(12,16)}$. The aim of the study was to investigate the association of a healthy eating score with depression in Chilean older adults.

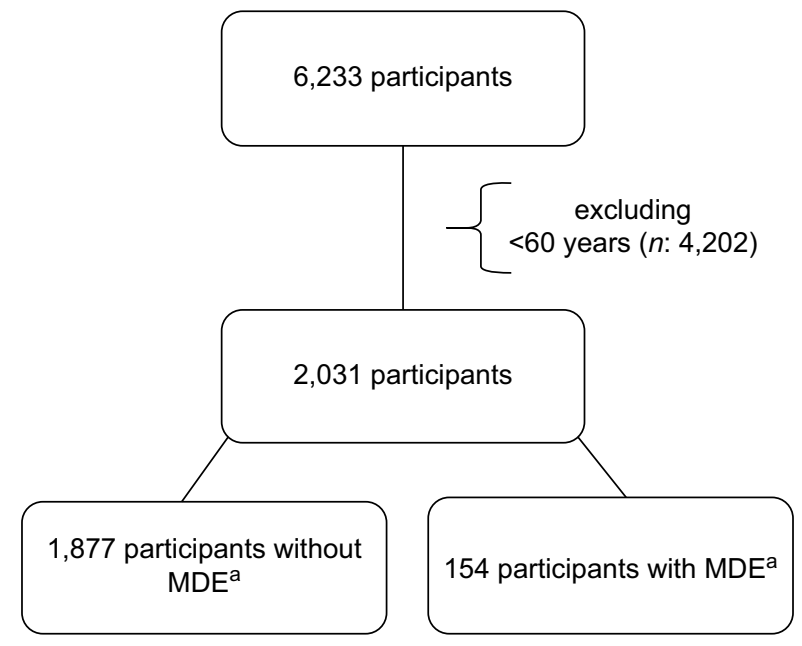

Fig. 1 Flowchart of older adult participants from the Chilean National Health Survey, 2016-2017. MDE = Major depressive episode

\section{Methods}

\section{Study design}

This cross-sectional study included older adults ( $\geq 60$ years) from the last Chilean National Health Survey (CNHS) conducted in 2016-2017 (2). The CNHS 2016-2017 included participants according to a stratified multistage sampling of non-institutionalised participants from urban and rural regions in Chile. Detailed information about the survey has been described elsewhere ${ }^{(17)}$. In the present study, from 6233 surveyed participants, 2031 subjects aged $\geq 60$ years were included in the analysis (Fig. 1).

\section{Major depressive episode}

The CIDI-SF was used to determine MDE. The CIDI-SF is a shorter version of a fully structured diagnostic interview designed for general population studies and was validated for the Chilean population ${ }^{(18,19)}$. Only the MDE module of the CIDI-SF was included to establish a diagnosis of MDE in the past 12 months. The Latin American translation of the latest version of the instrument was used, and minor changes were made in the language to adapt it to Spanish ${ }^{(20)}$.

Briefly, to determine a diagnosis of MDE, a minimum of five symptoms are required, out of which one has to be dysphoria or anhedonia ${ }^{(6)}$. The CIDI-SF has two stem questions administered to all subjects: (i) In the past 12 months, have you had 2 weeks or more when nearly every day you felt sad, blue or depressed?"; and (ii) In the past 12 months, have there been 2 weeks or longer when you lost interest in most things like work or hobbies or things you usually like to do for fun? Following the endorsement of one of the stem questions, more detailed questions on symptom intensity and duration are presented to the 
Table 1 Criteria for the healthy eating score

\begin{tabular}{|c|c|c|}
\hline Food group & $\begin{array}{l}\text { Frequency or intake of } \\
\text { consumption }\end{array}$ & Score \\
\hline Seafood & $\begin{array}{l}<1 \text { time/month or never } \\
1 \text { time/week or }<3 \text { times/month } \\
>1 \text { time/week }\end{array}$ & $\begin{array}{l}0 \\
1 \\
2\end{array}$ \\
\hline Whole grains & $\begin{array}{l}\text { Never } \\
1 \text { time/week or } 1 \text { time/month } \\
\geq 1 \text { portion/d or every } 2 \mathrm{~d}\end{array}$ & $\begin{array}{l}0 \\
1 \\
2\end{array}$ \\
\hline Dairy products & $\begin{array}{l}1 \text { time/month or never } \\
\text { Every } 2 \mathrm{~d} \text { or }>1 \text { time/week } \\
\geq 1 \text { portion/ }\end{array}$ & $\begin{array}{l}0 \\
1 \\
2\end{array}$ \\
\hline Fruits & $\begin{array}{l}<57 \mathrm{~g} / \mathrm{d} \\
57 \mathrm{~g}-137 \mathrm{~g} / \mathrm{d} \\
>137 \mathrm{~g} / \mathrm{d}\end{array}$ & $\begin{array}{l}0 \\
1 \\
2\end{array}$ \\
\hline Vegetable & $\begin{array}{l}<69 \mathrm{~g} / \mathrm{d} \\
69-137 \mathrm{~g} / \mathrm{d} \\
>137 \mathrm{~g} / \mathrm{d}\end{array}$ & $\begin{array}{l}0 \\
1 \\
2\end{array}$ \\
\hline Legumes & $\begin{array}{l}<1 \text { time/week or never } \\
1 \text { time/week }\end{array}$ & $\begin{array}{l}0 \\
1\end{array}$ \\
\hline Total score (adherence) & $\begin{array}{l}\geq 2 \text { portion/week } \\
\geq 9 \text { (healthy), } 5-8 \text { (average) } \\
\quad \text { and } \leq 4 \text { (unhealthy) }\end{array}$ & 2 \\
\hline
\end{tabular}

Healthy eating score was calculated by summing up the scores of six foods items of the Chilean National Health Survey, 2016-2017.

subject $^{(21)}$. The Survey assessed a total of eight symptoms of depression: dysphoria or anhedonia, fatigue, weight change, insomnia, problems of concentration, feelings of worthlessness and thoughts of death.

\section{Healthy eating score}

Consumption of six food items was included in the healthy eating habits: consumption of seafood, whole grain, dairy products, fruits, vegetables and legumes. These questions were designed by experts based on the food-based dietary guideline for the Chilean population as described in the CNHS 2009-2010 ${ }^{(17,20)}$. Frequency of consumption of seafood (How often do you consume fish and shell food?), whole grain (How often do you consume any whole grain products like whole grain bread, whole grain cereal or any other food that contains whole grain flour?), dairy products (How often do you consume milk, cheese, fresh cheese or yogurt?) and legumes (how often do you consume any type of legumes, such as beans, lentils, peas, chickpeas? The answers to these questions were scored according to the recommendations of national dietary guidelines ${ }^{(22)}$, from zero point for no compliance to two points for complete compliance, according to Table 1. For intake of fruits and vegetables, it was possible to calculate an average intake/d according to the dietary report of the survey. An intake of $80 \mathrm{~g} /$ serving was considered as one standard portion size of fruits or vegetables according to the Survey guidelines of use ${ }^{(17)}$. Participants were scored according to tertiles of intake as $\mathrm{g} / \mathrm{d}$. Consumption of fruits and vegetables was estimated through the questions: 'Typically, how many days a week do you eat fruits?', 'Typically, how many days a week do you eat vegetables or vegetable salad? (do not include legumes or potatoes)', and 'How many servings of fruits/vegetables or vegetable salad do you eat in one of those days? (supported by a deck of cards with standardised serving sizes)'. The healthy eating score ranged from 0 to 12 points and was categorised in three levels: healthy adherence to a healthy diet $(\geq 9)$, average adherence (5-8) and unhealthy adherence to a healthy $\operatorname{diet}(\leq 4)$.

\section{Socio-demographic, lifestyle variables and clinical covariates}

Socio-demographic variables, such as geographic area (urban and rural), age, sex, education level (elementary $<8$ years, secondary: $8-12$ years and higher education $>12$ years) and lifestyle characteristics, such as physical activity using the Global Physical Activity Questionnaire levels (low, moderate and high), hours of sleep ( $<7,7$ to 9 as a regular or $>9 \mathrm{~h} / \mathrm{d}$ ), tobacco status (current, former and never smokers) and alcohol consumption (more than 7 drinks/week, 1 to 7 drinks/week, less than 1 drink/week and none $)^{(23)}$, were self-reported and collected by trained interviewers. Chronic diseases such as hypertension (HT, self-reported, medication use or systolic/diastolic $\mathrm{BP}>140 / 90 \mathrm{mmHg}$ ), type 2 diabetes (self-reported or fasting blood glucose $>7 \mathrm{mmol} / \mathrm{l}$ ), hypercholesterolaemia (self-reported or treatment program) and previous CVD (self-reported acute myocardial infarction, stroke or peripheral artery disease) were also recorded. BMI was calculated from body weight divided by the square of the height and was classified using the following cut-off points: $<25,25-30$ and $>30 \mathrm{~kg} / \mathrm{m}^{2}$. Less than $1 \%$ of the participants showed a BMI $<18.5 \mathrm{~kg} / \mathrm{m}^{2}$ and they were included within the BMI category $<25.0 \mathrm{~kg} / \mathrm{m}^{2}$. Finally, a score below 13 on the Mini-Mental State Examination was used to define cognitive impairment ${ }^{(24)}$. All clinical measures were conducted by trained nurses. Before blood pressure measurement, individuals were instructed to remain seated and calm for $5 \mathrm{~min}$. Blood pressure was measured using the Omron HEM $742^{\circledR}$ BP monitor and derived from the mean of three readings recorded ( $2 \mathrm{~min}$ between measures). Blood extraction was carried out according to the Survey manual and blood analyses were performed in a centralised, internationally certified clinical laboratory. Weight measurement was carried out with a digital scale (Tanita HD-313 ${ }^{\circledR}$ ) and height with a rod in the individuals' home, following the protocols in $\mathrm{CNHS}^{(17)}$. Participants were particularly instructed not to wear shoes and to dress in light clothes.

\section{Statistical analyses}

Descriptive statistics included mean and $95 \%$ CI for continuous variables, and percentage or frequency and $95 \%$ CI for categorical variables. The analyses were weighed 
by the characteristics of the Chilean population and the CNHS design. Differences in healthy eating score stratified by socio-demographic, lifestyles and clinical conditions were compared using complex samples general linear models adjusted for age, sex and geographical area.

The association of the healthy eating score with MDE was assessed using complex samples logistic regression models. The results are presented as odds ratio (OR) with their $95 \%$ CI. Participants with the unhealthiest healthy eating score were defined as the reference group. All analyses were adjusted for potential confounders using three statistical models: model 1-adjusted for age, sex, region, and residency area (urban and rural), and education level; model 2-additionally adjusted for tobacco status, alcohol intake and physical activity and model 3-further adjusted for BMI, cognitive impairment, hypertension, hypercholesterolaemia, diabetes and previous CVD. In addition, we analysed the association between each of the individual food items of the healthy eating score and MDE in model 3 (excluding the healthy eating score). The percent contribution of each food group to the effect of the healthy eating score on MDE was calculated including each food item separately in model 3 following the formula (OR base model OR adjusted model)/(OR base model -1$) \times 100 \%(25,26)$

Sensitivity analysis excluding participants with data imputations (BMI (12.8\%); alcohol consumption (10.0\%); physical activity (3.1\%); educational level $(1.3 \%)$ and $\operatorname{CVD}(0.2 \%))$, living in rural areas, having a BMI $<18.5 \mathrm{~kg} / \mathrm{m}^{2}$, participants with risky alcohol consumption (more than 7 drinks/week), patients with diabetes, previous CVD and cognitive impairment were conducted. Analysis for confounding variables and healthy eating score and their interactions were also performed. The significance level was defined as $P<0.05$. IBM SPSS 27.0 was used for statistical analysis.

\section{Results}

General characteristics according to healthy eating score categories are shown in Table 2. In the descriptive data for sociodemographic, it can be noted that participants with unhealthy eating score had lower education levels and were more likely to reside in rural areas than participants with average or with a better healthy eating score. Furthermore, participants with an unhealthiest healthy eating score showed lower physical activity levels, regular hours of sleep and lower prevalence of hypercholesterolemia than those with a healthiest healthy eating score. (Table 2). There was a non-statistically significant trend towards a higher prevalence of previous CVD, cognitive impairment and depression in subjects with an unhealthier or average score than in those with the healthiest eating score.

The association between depression and the healthy eating score is shown in Table 3. Participants with a healthy $v$. unhealthy eating score showed a lower risk for depression (OR: 0.280 (95\% CI 0.104, 0.749)). No statistically significant association was observed in participants with an average healthy eating score compared with the unhealthy eating score.

Results of the fully adjusted model are shown in Fig. 2. Apart from the healthy eating score, previous CVD $(P<0.001)$ and hours of sleep $<7 \mathrm{~h}(P=0.002)$ were positively associated with depression. When the healthy eating score items were evaluated in isolation in the full adjusted model, there was a statistically significant negative association between legume consumption and $\operatorname{MDE}(P=0.046)$, and a positive one with vegetables intake $(P=0.018$; Fig. 3 : Panel A). In addition, the contribution of each food group to the effect of the total score on depression was calculated (Fig. 3, Panel B). The most important food items that contributed to the association of the healthy eating score with depression were legumes (15.2\%) and seafood (12.7\%). Conversely, the items of vegetables $(-20.6 \%)$ and fruits $(-8.4 \%)$ had a negative impact on the association between the healthy eating score and depression (Fig. 3, Panel B).

Sensitivity analyses are shown in Supplemental Table 1. Excluding participants with data imputation, with underweight (BMI $<18.5 \mathrm{~kg} / \mathrm{m}^{2}$ ), with risky alcohol consumption (more than 7 drinks/week), previous CVD or cognitive impairment showed similar results for the association between healthy eating index and depression (see online Supplemental Table 1). However, after excluding participants from rural areas and with diabetes, the statistical significance was lost. There was a statistically significant interaction of the healthy eating score with living area and educational level for MDE $(P<0.001)$. Results remained similar for participants living in urban areas (OR healthy $v$. unhealthy: $0.339,(95 \% \mathrm{CI} 0 \cdot 112,1.023)$, and with a low and middle educational level ( $\mathrm{OR}_{\text {healthy }} v$. unhealthy: 0.673, (95\% CI 0.220, 2.058); OR healthy $v$. unhealthy: 0.02, (95\% CI 0.01, 0.20), respectively). However, among subjects living in rural areas or with a high educational level, there was a very low number of participants with MDE scoring in the different categories of the healthy eating score and calculations were not possible. There were no other statistically significant interactions between the healthy eating score and the covariates of the study for MDE.

\section{Discussion}

The present study confirms that high adherence to a healthy dietary pattern is associated with a reduced risk for depression in a national representative sample of older adults from Chile. The food items within the healthy eating score that contributed the most to this association were the consumption of legumes and seafood. Similar results were observed in previous studies conducted in different samples from Latin American countries, but not at a national 


\begin{tabular}{|c|c|c|c|c|c|c|c|c|c|}
\hline \multirow[b]{2}{*}{ Variablesł } & \multirow[b]{2}{*}{ All \% } & \multirow[b]{2}{*}{$95 \% \mathrm{Cl}$} & \multicolumn{6}{|c|}{ Healthy eating score } & \multirow[b]{2}{*}{$P_{\text {for trend }}{ }^{*}$} \\
\hline & & & Unhealthy & $95 \% \mathrm{Cl}$ & Average & $95 \% \mathrm{Cl}$ & Healthy & $95 \% \mathrm{Cl}$ & \\
\hline Participants $(n)$ & 2031 & & 576 & & 1180 & & 275 & & \\
\hline Weighed population $(n)$ & 3066213 & & 744274 & & 1868418 & & 453520 & & \\
\hline Prevalence & 100 & & 24.3 & $21 \cdot 2,27 \cdot 7$ & 60.9 & $57 \cdot 1,64 \cdot 7$ & $14 \cdot 8$ & $12 \cdot 0,18 \cdot 1$ & \\
\hline Sex & & & & & & & & & 0.113 \\
\hline Men & $48 \cdot 2$ & $44 \cdot 2,52 \cdot 3$ & 48.4 & $40 \cdot 8,56 \cdot 1$ & 51.5 & $46 \cdot 2,56 \cdot 7$ & 34.5 & $23 \cdot 9,46.9$ & \\
\hline Women & $51 \cdot 8$ & $47 \cdot 7,55 \cdot 8$ & 51.6 & $43 \cdot 9,59 \cdot 2$ & 48.5 & $43 \cdot 3,53 \cdot 8$ & 65.5 & $53 \cdot 1,76 \cdot 1$ & \\
\hline Age (years) & 69.5 & $68 \cdot 8,70 \cdot 2$ & $69 \cdot 7$ & $68.5,71.0$ & 69.5 & $68.5,70 \cdot 6$ & 69.5 & $67 \cdot 8,70 \cdot 6$ & 0.552 \\
\hline Area & & & & & & & & & $<0.001$ \\
\hline Rural & 14.0 & $11 \cdot 8,16 \cdot 6$ & 21.0 & $16 \cdot 6,26 \cdot 2$ & $12 \cdot 0$ & $9 \cdot 5,15 \cdot 0$ & $10 \cdot 7$ & $6 \cdot 7,16 \cdot 9$ & \\
\hline Urban & $86 \cdot 0$ & $83 \cdot 4,88 \cdot 2$ & $79 \cdot 0$ & $73 \cdot 8,83 \cdot 4$ & $88 \cdot 0$ & $85 \cdot 0,90 \cdot 5$ & $89 \cdot 3$ & $83 \cdot 1,93 \cdot 3$ & \\
\hline Educational levelł & & & & & & & & & $<0.001$ \\
\hline$<8$ years & $46 \cdot 9$ & $42 \cdot 0,51 \cdot 8$ & $61 \cdot 7$ & $53 \cdot 6,69 \cdot 2$ & 43.9 & $37 \cdot 8,50 \cdot 2$ & 34.8 & $24 \cdot 2,47 \cdot 0$ & \\
\hline $8-12$ years & $36 \cdot 9$ & $32 \cdot 5,41 \cdot 6$ & 29.5 & $22 \cdot 5,37 \cdot 7$ & $39 \cdot 6$ & $33 \cdot 8,45 \cdot 8$ & $38 \cdot 1$ & $28 \cdot 3,48.9$ & \\
\hline$>12$ years & $16 \cdot 2$ & $12 \cdot 2,21 \cdot 2$ & 8.8 & $5 \cdot 1,14 \cdot 8$ & $16 \cdot 5$ & $11.5,23.0$ & $27 \cdot 2$ & $17 \cdot 6,39 \cdot 5$ & \\
\hline BMI category $\ddagger$ & & & & & & & & & 0.231 \\
\hline$<25 \mathrm{~kg} / \mathrm{m}^{2}$ & $18 \cdot 3$ & $15 \cdot 5,21 \cdot 6$ & 19.5 & $14 \cdot 8,25 \cdot 4$ & $19 \cdot 2$ & $15 \cdot 2,24 \cdot 0$ & $12 \cdot 7$ & $8 \cdot 3,19 \cdot 0$ & \\
\hline $25-30 \mathrm{~kg} / \mathrm{m}^{2}$ & 44.7 & $40.1,49.5$ & 44.9 & $37 \cdot 5,52 \cdot 6$ & $45 \cdot 0$ & $39 \cdot 1,51 \cdot 1$ & $43 \cdot 1$ & $32 \cdot 8,54 \cdot 1$ & \\
\hline$\geq 30 \mathrm{~kg} / \mathrm{m}^{2}$ & $36 \cdot 9$ & $32 \cdot 7,41 \cdot 4$ & 35.5 & $28 \cdot 9,42 \cdot 7$ & $35 \cdot 8$ & $30 \cdot 3,41 \cdot 6$ & $44 \cdot 2$ & $33 \cdot 4,55 \cdot 6$ & \\
\hline Lifestyles & & & & & & & & & \\
\hline Physical activity $\ddagger$ & & & & & & & & & 0.005 \\
\hline Low & $45 \cdot 6$ & $41 \cdot 5,49 \cdot 7$ & 52.4 & $44 \cdot 9,59 \cdot 7$ & 44.0 & $38.8,49.4$ & $40 \cdot 9$ & $30 \cdot 2,52 \cdot 5$ & \\
\hline Moderate & $25 \cdot 6$ & $22 \cdot 0,29 \cdot 6$ & $28 \cdot 2$ & $21 \cdot 4,36 \cdot 2$ & $25 \cdot 6$ & $21 \cdot 0,30 \cdot 7$ & 21.5 & $14 \cdot 1,31 \cdot 2$ & \\
\hline High & $28 \cdot 8$ & $24 \cdot 9,33 \cdot 1$ & $19 \cdot 4$ & $14 \cdot 9,25 \cdot 0$ & $30 \cdot 4$ & $25.4,35.9$ & $37 \cdot 7$ & $27 \cdot 0,49 \cdot 7$ & \\
\hline Tobacco status & & & & & & & & & 0.930 \\
\hline Never & $46 \cdot 1$ & $42 \cdot 2,50 \cdot 0$ & $47 \cdot 4$ & $40 \cdot 3,54 \cdot 6$ & $45 \cdot 4$ & $40 \cdot 2,50 \cdot 6$ & $46 \cdot 7$ & $36 \cdot 2,57 \cdot 5$ & \\
\hline Former & $40 \cdot 7$ & $36 \cdot 5,45 \cdot 0$ & $41 \cdot 1$ & $33 \cdot 6,49 \cdot 1$ & 39.9 & $34 \cdot 6,45 \cdot 4$ & 43.4 & $32 \cdot 0,55 \cdot 4$ & \\
\hline Current & $13 \cdot 3$ & $10 \cdot 7,16 \cdot 3$ & 11.5 & $7 \cdot 6,16 \cdot 9$ & 14.8 & $11 \cdot 1,19 \cdot 4$ & 9.9 & $5 \cdot 3,17.9$ & \\
\hline Alcoholł & & & & & & & & & 0.207 \\
\hline None & 41.5 & $37 \cdot 5,45 \cdot 7$ & 44.9 & $37 \cdot 7,52 \cdot 3$ & $39 \cdot 1$ & $34 \cdot 1,44.5$ & 45.9 & $34 \cdot 6,57 \cdot 6$ & \\
\hline Less than 1.0 drink $p / w$ & $28 \cdot 9$ & $25 \cdot 3,32 \cdot 8$ & $28 \cdot 7$ & $22 \cdot 7,35 \cdot 6$ & $30 \cdot 1$ & $25 \cdot 2,35 \cdot 5$ & $24 \cdot 1$ & $17 \cdot 0,33 \cdot 0$ & \\
\hline 1.0 to 7.0 drinks $p / w$ & $21 \cdot 1$ & $17 \cdot 8,24 \cdot 9$ & $20 \cdot 0$ & $13 \cdot 6,28 \cdot 2$ & 22.4 & $18 \cdot 2,27 \cdot 2$ & $17 \cdot 6$ & $11 \cdot 1,26 \cdot 8$ & \\
\hline More than 7.0 drinks $\mathrm{p} / \mathrm{w}$ & 8.5 & $6 \cdot 0,12 \cdot 0$ & 6.4 & $3 \cdot 3,12 \cdot 0$ & 8.4 & $5 \cdot 3,13 \cdot 0$ & 12.4 & $5 \cdot 2,26 \cdot 8$ & \\
\hline Hours of sleep & & & & & & & & & $<0.001$ \\
\hline$<7$ & $42 \cdot 0$ & $38 \cdot 0,46 \cdot 1$ & $53 \cdot 3$ & $45 \cdot 9,60 \cdot 6$ & $53 \cdot 8$ & $48 \cdot 7,58 \cdot 8$ & $40 \cdot 3$ & $31 \cdot 0,50 \cdot 3$ & \\
\hline $7-9$ & $51 \cdot 7$ & $47 \cdot 7,55 \cdot 7$ & $36 \cdot 0$ & $29 \cdot 2,43.5$ & $41 \cdot 1$ & $36 \cdot 1,46 \cdot 3$ & $55 \cdot 6$ & $45 \cdot 1,65 \cdot 5$ & \\
\hline$>9$ & 6.3 & $4 \cdot 9,8 \cdot 1$ & $10 \cdot 6$ & $7 \cdot 4,15 \cdot 1$ & $5 \cdot 1$ & $3.6,7 \cdot 2$ & 4.2 & $2 \cdot 1,8 \cdot 4$ & \\
\hline Healthy eating habits§ & & & & & & & & & \\
\hline Seafood & & & & & & & & & $<0.001$ \\
\hline$<1$ time $\mathrm{p} / \mathrm{m}$ or never & $29 \cdot 9$ & $26 \cdot 6,33 \cdot 5$ & $52 \cdot 9$ & $45 \cdot 1,60 \cdot 6$ & $26 \cdot 2$ & $21 \cdot 8,31 \cdot 1$ & $7 \cdot 6$ & $3 \cdot 9,14 \cdot 1$ & \\
\hline $1.0 \mathrm{p} / \mathrm{w}$ or $<3$ times $\mathrm{p} / \mathrm{m}$ & $61 \cdot 0$ & $56 \cdot 8,65 \cdot 0$ & $45 \cdot 8$ & $38 \cdot 1,53 \cdot 7$ & $65 \cdot 1$ & $59 \cdot 7,70 \cdot 2$ & $68 \cdot 8$ & $57.5,78.3$ & \\
\hline$>1.0 \mathrm{p} / \mathrm{w}$ & 9.1 & $6 \cdot 6,12 \cdot 3$ & 1.3 & $0.6,2.5$ & 8.7 & $5 \cdot 4,13 \cdot \overline{7}$ & $23 \cdot 6$ & $15 \cdot 9,33 \cdot 6$ & \\
\hline Whole grain & & & & & & & & & $<0.001$ \\
\hline Never & 63.9 & $59 \cdot 6,68 \cdot 0$ & 85.4 & $79 \cdot 4,89.9$ & 65.9 & $60 \cdot 3,71 \cdot 2$ & $20 \cdot 1$ & $11.4,32.9$ & \\
\hline 1 time $p / w$ or 1 time $p / m$. & $17 \cdot 8$ & $15 \cdot 2,20 \cdot 8$ & $10 \cdot 9$ & $7 \cdot 5,15 \cdot 7$ & 21.5 & $17 \cdot 6,25 \cdot 9$ & $14 \cdot 3$ & $9 \cdot 0,21.9$ & \\
\hline$\geq 1 \mathrm{p} / \mathrm{d}$ or every $2 \mathrm{~d}$ & $18 \cdot 3$ & $15 \cdot 2,21 \cdot 8$ & 3.6 & $1.4,9.4$ & 12.6 & $9 \cdot 3,16 \cdot 9$ & 65.6 & $53 \cdot 6,76 \cdot 0$ & \\
\hline
\end{tabular}




\section{NS Public Health Nutrition}

Table 2 Continued

\begin{tabular}{|c|c|c|c|c|c|c|c|c|c|}
\hline \multirow[b]{2}{*}{ Variables $\ddagger$} & \multirow[b]{2}{*}{ All \% } & \multirow[b]{2}{*}{$95 \% \mathrm{Cl}$} & \multicolumn{6}{|c|}{ Healthy eating score } & \multirow[b]{2}{*}{$P_{\text {for trend }}{ }^{*}$} \\
\hline & & & Unhealthy & $95 \% \mathrm{Cl}$ & Average & $95 \% \mathrm{Cl}$ & Healthy & $95 \% \mathrm{Cl}$ & \\
\hline Dairy products & & & & & & & & & $<0.001$ \\
\hline 1 time $\mathrm{p} / \mathrm{m}$ or never & 11.5 & $9 \cdot 2,14 \cdot 2$ & $26 \cdot 8$ & $20 \cdot 9,33 \cdot 6$ & $7 \cdot 8$ & $5 \cdot 4,11 \cdot 2$ & $1 \cdot 2$ & $0.2,5.9$ & \\
\hline Every $2 \mathrm{~d}$ or $>1$ time $\mathrm{p} / \mathrm{w}$ & $35 \cdot 8$ & $31 \cdot 8,40 \cdot 0$ & $54 \cdot 3$ & $46 \cdot 8,61 \cdot 6$ & $32 \cdot 9$ & $28 \cdot 3,37.9$ & $17 \cdot 2$ & $10 \cdot 1,27 \cdot 9$ & \\
\hline$\geq 1 \mathrm{p} / \mathrm{d}$ & $52 \cdot 8$ & $48 \cdot 7,56 \cdot 8$ & $19 \cdot 0$ & $14 \cdot 7,24 \cdot 1$ & $59 \cdot 2$ & $54 \cdot 2,64 \cdot 1$ & 81.6 & $70 \cdot 9,88.9$ & \\
\hline Fruits & & & & & & & & & $<0.001$ \\
\hline $\operatorname{Low}(\leq 57 \mathrm{~g} / \mathrm{d})$ & $29 \cdot 1$ & $25 \cdot 2,33 \cdot 2$ & 64.4 & $56 \cdot 6,71 \cdot 5$ & $21 \cdot 8$ & $16 \cdot 9,27 \cdot 7$ & 1.0 & $0.3,3.2$ & \\
\hline Medium $(57 \mathrm{~g}-137 \mathrm{~g} / \mathrm{d})$ & $32 \cdot 9$ & $29 \cdot 3,36 \cdot 7$ & $28 \cdot 5$ & $22 \cdot 0,36 \cdot 0$ & $38 \cdot 2$ & $33 \cdot 3,43.5$ & $17 \cdot 9$ & $12 \cdot 1,25 \cdot 8$ & \\
\hline High ( $\geq 137 \mathrm{~g} / \mathrm{d})$ & $38 \cdot 0$ & $34 \cdot 0,42 \cdot 3$ & $7 \cdot 1$ & $3 \cdot 9,12 \cdot 6$ & 39.9 & $34 \cdot 8,45 \cdot 2$ & $81 \cdot 1$ & $72 \cdot 9,87 \cdot 2$ & \\
\hline Fruits $(g / d)$ & $126 \cdot 1$ & $114 \cdot 7,137 \cdot 5$ & $71 \cdot 0$ & $48 \cdot 0,94 \cdot 0$ & $127 \cdot 7$ & $114 \cdot 2,141 \cdot 2$ & $210 \cdot 1$ & $187 \cdot 2,233 \cdot 0$ & \\
\hline Vegetables & & & & & & & & & $<0.001$ \\
\hline Low $(\leq 69 \mathrm{~g} / \mathrm{d})$ & $24 \cdot 1$ & $20 \cdot 9,27 \cdot 6$ & $65 \cdot 6$ & $58 \cdot 6,71 \cdot 9$ & $13 \cdot 1$ & $10 \cdot 2,16 \cdot 5$ & 1.4 & $0.5,4.0$ & \\
\hline Medium $(69-137 \mathrm{~g} / \mathrm{d})$ & $35 \cdot 6$ & $31 \cdot 6,59 \cdot 7$ & 29.5 & $23 \cdot 5,36 \cdot 3$ & $40 \cdot 9$ & $35 \cdot 7,46 \cdot 3$ & $23 \cdot 8$ & $15 \cdot 4,34 \cdot 8$ & \\
\hline $\operatorname{High}(\geq 137 \mathrm{~g} / \mathrm{d})$ & $40 \cdot 3$ & $36 \cdot 0,44 \cdot 8$ & $5 \cdot 0$ & $2 \cdot 9,8 \cdot 4$ & $46 \cdot 1$ & $40 \cdot 6,51 \cdot 6$ & 74.8 & $63 \cdot 7,83 \cdot 4$ & \\
\hline Vegetables $(g / d)$ & $127 \cdot 7$ & $105 \cdot 8,149 \cdot 5$ & 59.4 & $54 \cdot 2,64 \cdot 7$ & 144.8 & $109 \cdot 8,179 \cdot 9$ & 168.9 & $154 \cdot 4,183 \cdot 4$ & \\
\hline Legumes & & & & & & & & & $<0.001$ \\
\hline$<1$ time/week or never & $18 \cdot 2$ & $15 \cdot 2,21 \cdot 6$ & 38.4 & $31 \cdot 7,45 \cdot 7$ & 14.5 & $10 \cdot 6,19 \cdot 6$ & 0.3 & $0 \cdot 1,1 \cdot 1$ & \\
\hline 1 time/week & $51 \cdot 1$ & $46 \cdot 7,55 \cdot 5$ & $47 \cdot 6$ & $40 \cdot 5,54.9$ & $55 \cdot 3$ & $49 \cdot 5,60 \cdot 9$ & $39 \cdot 8$ & $29 \cdot 5,51 \cdot 0$ & \\
\hline$\geq 2 \mathrm{p} / \mathrm{w}$ & $30 \cdot 6$ & $26 \cdot 7,34 \cdot 8$ & $13 \cdot 9$ & $8.5,21.9$ & $30 \cdot 2$ & $25 \cdot 0,35 \cdot 8$ & 59.9 & $48 \cdot 7,70 \cdot 2$ & \\
\hline $\begin{array}{l}\text { Healthy eating score } \\
\text { (units) }\end{array}$ & $6 \cdot 1$ & $5 \cdot 9,6 \cdot 3$ & 3.1 & $3 \cdot 0,3 \cdot 2$ & $6 \cdot 4$ & $6 \cdot 3,6 \cdot 6$ & $9 \cdot 5$ & $9 \cdot 3,9 \cdot 7$ & $<0.001$ \\
\hline Chronic diseases & & & & & & & & & \\
\hline Hypertension, yes & $72 \cdot 3$ & $68 \cdot 9,76 \cdot 2$ & $75 \cdot 1$ & $68 \cdot 5,80 \cdot 8$ & 74.5 & $69 \cdot 7,78 \cdot 8$ & $61 \cdot 5$ & $49 \cdot 9,71.9$ & 0.063 \\
\hline Diabetes, yes & $29 \cdot 6$ & $25 \cdot 7,33.9$ & $24 \cdot 3$ & $18 \cdot 6,31 \cdot 2$ & $31 \cdot 0$ & $25 \cdot 6,37 \cdot 0$ & $32 \cdot 7$ & $23 \cdot 4,43.5$ & 0.192 \\
\hline Hypercholesterolemia, yes & $41 \cdot 3$ & $37 \cdot 2,45 \cdot 5$ & 34.8 & $28 \cdot 3,42 \cdot 0$ & $40 \cdot 8$ & $35 \cdot 4,46 \cdot 4$ & $54 \cdot 0$ & $42 \cdot 5,65 \cdot 1$ & 0.010 \\
\hline CVDł, yes & 23.9 & $20 \cdot 4,27 \cdot 8$ & $26 \cdot 7$ & $20 \cdot 2,34 \cdot 4$ & $23 \cdot 8$ & $19 \cdot 2,29 \cdot 1$ & $19 \cdot 7$ & $13 \cdot 8,27 \cdot 4$ & 0.116 \\
\hline Mild cognitive impairment, yes & 9.9 & $7 \cdot 8,12 \cdot 5$ & $12 \cdot 6$ & $8 \cdot 3,18 \cdot 8$ & $9 \cdot 6$ & $7 \cdot 2,12 \cdot 7$ & $6 \cdot 9$ & $3 \cdot 7,12 \cdot 6$ & 0.195 \\
\hline Depression, yes & 7.9 & $6 \cdot 2,10 \cdot 1$ & $8 \cdot 3$ & $5 \cdot 0,13 \cdot 5$ & $9 \cdot 1$ & $6 \cdot 7,12 \cdot 3$ & $2 \cdot 3$ & $1 \cdot 1,4 \cdot 6$ & 0.729 \\
\hline
\end{tabular}

${ }^{*}$ Differences in socio-demographic, lifestyles and clinical conditions were compared across healthy eating score categories using complex samples general lineal models adjusted for age, sex and geographical area $(P<0.05)$. †Variables were reported as number of subjects, mean values and $95 \% \mathrm{Cl}$, or percentages and $95 \% \mathrm{Cl}$.

ҒAvailable data for the following variables are (n): educational level (2005); BMI (1773); physical activity (1969); alcohol (1826) and CVD (2027).

§p/d: portion per day; p/w: portion per week; p/m: portion per month. 
Table 3 Association between healthy eating score and major depression episode risk in older adults ( $\geq 60$ years) from the Chilean National Health Survey, 2016-2017

\begin{tabular}{|c|c|c|c|c|c|c|c|c|c|c|c|}
\hline \multirow{2}{*}{$\begin{array}{l}\text { Healthy eating } \\
\text { score }\end{array}$} & \multicolumn{2}{|c|}{$\begin{array}{c}\text { Unhealthy } \\
\text { (n 576, weighed } \\
\text { cases }=744274)\end{array}$} & \multicolumn{3}{|c|}{$\begin{array}{l}\text { Average }(n 1180, \text { weighed } \\
\text { cases }=1868418)\end{array}$} & \multicolumn{3}{|c|}{$\begin{array}{l}\text { Healthy }(n 275 \text {, weighed } \\
\text { cases }=453520)\end{array}$} & \multicolumn{3}{|c|}{$\begin{array}{l}\text { Per unit increase in } \\
\text { healthy eating score }\end{array}$} \\
\hline & OR & $95 \% \mathrm{Cl}$ & OR & $95 \% \mathrm{Cl}$ & $P$ & OR & $95 \% \mathrm{Cl}$ & $P$ & OR & $95 \% \mathrm{Cl}$ & $P$ \\
\hline Model 1 & 1 & Ref & $1 \cdot 12$ & $0.58,2.16$ & 0.719 & 0.25 & $0.10,0.65$ & 0.004 & 0.71 & $0.49,1.01$ & 0.061 \\
\hline Model 2 & 1 & Ref & $1 \cdot 12$ & $0 \cdot 60,2 \cdot 11$ & 0.737 & 0.28 & $0.10,0.75$ & 0.011 & 0.73 & $0.51,1.05$ & 0.095 \\
\hline Model 3 & 1 & Ref & $1 \cdot 14$ & $0.63,2.05$ & 0.691 & 0.28 & $0.10,0.74$ & 0.011 & 0.72 & $0.50,1.00$ & 0.064 \\
\hline
\end{tabular}

Model 1 was adjusted for age, sex, region, residency area and education level; model 2-additionally adjusted for tobacco status, alcohol intake, physical activity and hours of sleep; and model 3-additionally adjusted for BMI, cognitive impairment, hypertension, hypercholesterolaemia, diabetes and previous CVD (acute myocardial infarction, stroke or peripheral artery disease).

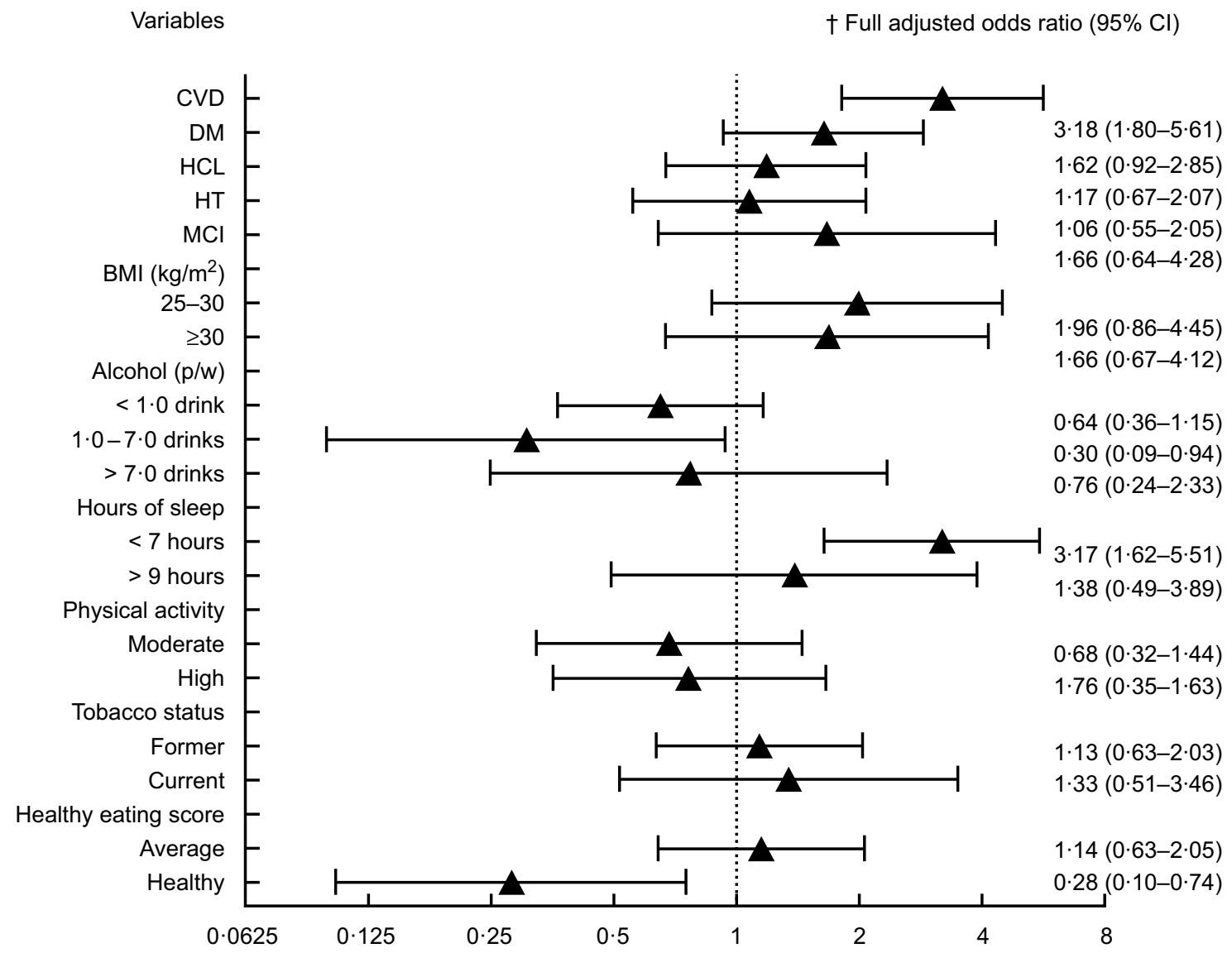

Fig. 2 Association between Major Depression Episode and lifestyles and clinical conditions among 2031 older adults ( $\geq 60$ years) from the Chilean National Health Survey, 2016-2017. CVD = cardiovascular diseases; DM =Diabetes mellitus; $\mathrm{HCL}=$ Hypercholesterolemia; $\mathrm{HT}=$ hypertension; $\mathrm{MCl}=$ mild Cognitive Impairment. Data is presented as $\mathrm{OR}$ and $95 \%$ of $\mathrm{Cl}$ $(95 \% \mathrm{Cl})$ for major depression episode. 'Logistic regression model included sex, region, residency area (urban and rural), education level, healthy eating score, tobacco status, alcohol intake, physical activity, hours of sleep, BMl, cognitive impairment, cardiovascular diseases, hypertension, hypercholesterolemia and diabetes

level $^{(14)}$. Up to our knowledge, few studies evaluated the association between diet and depression risk in older Latin American populations ${ }^{(14)}$. However, a healthier diet quality was associated with better global cognition and verbal skills in an older Hispanics/Latinos population ${ }^{(27)}$.

The National Survey of Food Consumption in Chile showed that only $5 \%$ of the Chilean population followed a healthy diet as defined by the Food-Based Dietary Guideline for the Chilean population ${ }^{(28)}$. Especially in the population aged $\geq 65$ years, low compliance for fruits and vegetables (51.4\%), dairy (19.4\%), legumes (29.0\%) and fish (13\%) were noticed. The last CNHS verifies even lower compliance in this age group regarding fruits and vegetables, fish and legumes intake ${ }^{(17)}$. In addition, 

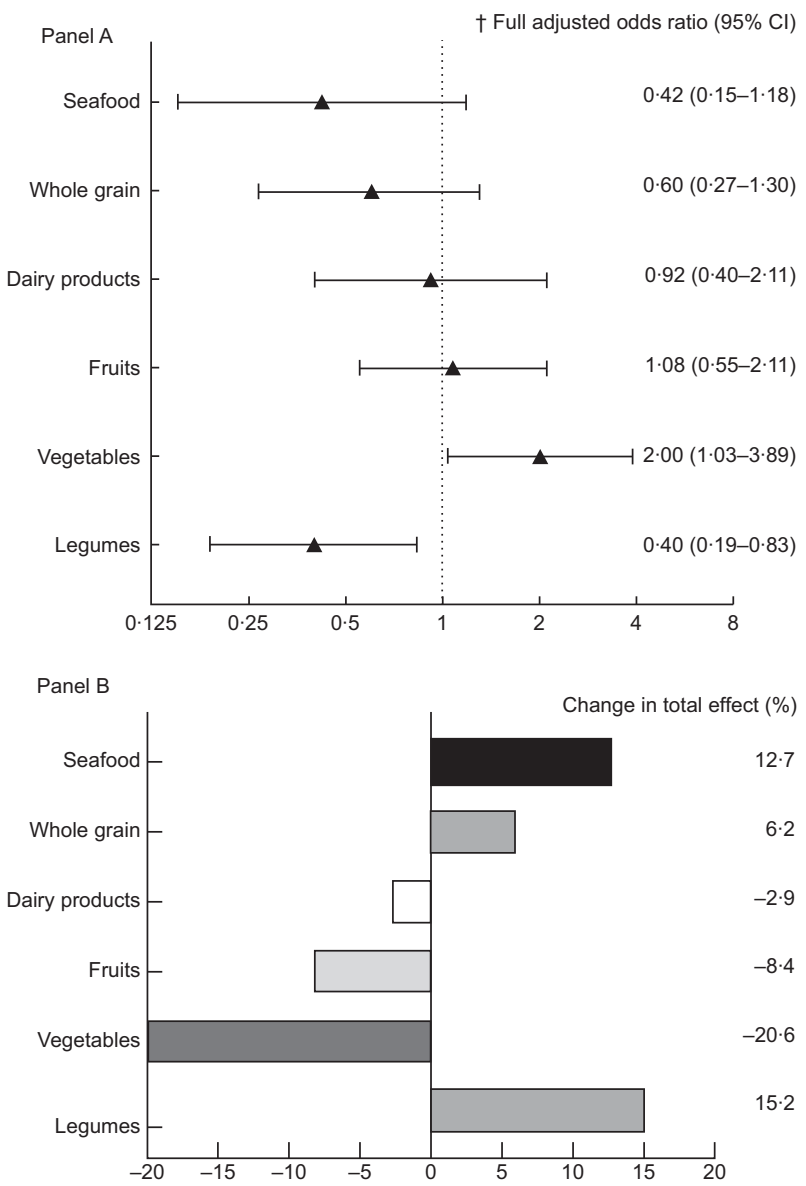

Fig. 3 Odds ratio for Major Depressive Episode (highest $v$. the lowest score) of each individual food item (Panel A), and percentage of change in total effect of the healthy eating score after controlling for each food group (Panel B), among 2031 older adults ( $\geq 60$ years) from the Chilean National Health Survey, 2016-2017. The percentage of change for healthy (high $v$. unhealthy) eating score after controlling for each food group was calculated using the formula (OR base model - OR adjusted model)/ (OR base model - 1) $\times 100 \%$. 'Model adjusted for age, sex, region, residency area, education, tobacco status, alcohol intake, physical activity, hours of sleep, BMI, cognitive impairment, hypertension, hypercholesterolemia, diabetes, cardiovascular diseases (acute myocardial infarction, stroke or peripheral artery disease), and the healthy eating score and each individual food item (as appropriate, for calculations of $\%$ of change in total effect)

multiple factors could influence eating behaviour in older adults increasing the barriers to fulfill the guidelines recommendations. Among them, chewing swallowing thirst, hunger, smell and taste may be altered as a consequence of age, as well as increased prevalence of gastrointestinal disorders $^{(29)}$. The latter might impact appetite, energy balance, weight control and critical micronutrient deficiencies, as it was found in a Chilean study ${ }^{(30)}$. Other socio-demographic, lifestyle and behavioural factors (e.g. family and social integration) have also been studied for their association with food consumption in older Chilean adults ${ }^{(31,32)}$.

Legumes were the food item that contributed the most to the association between the healthy eating score and depression risk, followed by seafood. Potential mechanisms between intake of legumes and depression include its content of fibre, zinc and protein, as well as its protein quality, with a relatively high content of tryptophan ${ }^{(33)}$. Legumes could play a role in depression through their contribution of dietary fibre and tryptophan; thus, improving the profile of gut microbiota and modulating tryptophan metabolism ${ }^{(34,35)}$. Tryptophan is precursor to serotonin, a neurotransmitter that is involved-among other functions-with mood regulation ${ }^{(36)}$. However, $90 \%$ of tryptophan catabolism occurs through the kynurenine pathway, while only approximately $3 \%$ of dietary tryptophan is involved in serotonin synthesis ${ }^{(37)}$. It is possible that legumes intake may alter this conversion ratio leading to a lower risk for chronic diseases ${ }^{(37)}$. Last, legumes are rich in micronutrients, especially zinc, which is another micronutrient related to depression ${ }^{(38,39)}$. In a recent meta-analysis, zinc supplementation was demonstrated as an effective adjunct therapy to anti-depressant medication $^{(40,41)}$.

From animal-derived foods, one of the top sources of tryptophan in the human diet is fish. In addition, seafood consumption could be related to depression by means of its high contribution of dietary PUFA. High intake of PUFA, and especially from the $\omega-3$ fatty acid series, was associated with reduced inflammation and better cognitive outcomes ${ }^{(42,43)}$. Although several randomised trials support the positive role of $n$ - 3 fatty acid supplementation in older patients with depression ${ }^{(44,45)}$, there are limited controlled studies ${ }^{(46)}$. Observational studies suggest a $\mathrm{U}$ or J-shape relationship between fish consumption and depression $^{(43)}$.

The counterintuitive results regarding the association between the consumption of fruits and vegetables, and depression risk may indicate errors in the method to estimate the dietary intake of these food groups. In fact, a meta-analysis provided evidence that fruit and vegetable intake was inversely associated with depression risk $^{(47)}$. Plant foods are rich sources of fibre, vitamins, minerals, antioxidants and phytochemicals. Indeed, a recent study found an inverse association between the consumption of fruits and vegetables and the risk for depressive symptoms ${ }^{(48)}$. Moreover, they showed that 3-indoxylsulfate levels could reflect the role of fibres in indole production associated with gut bacterial metabolism of tryptophan ${ }^{(48)}$. Also, lower consumption of fruits and vegetables has been associated with greater oxidative stress, which is related to elevated risk for mental health disorders ${ }^{(49)}$. Fruit and vegetable intake in the CNHS were reported in a different way than the other food items. Thus, the unexpected association between the consumption of fruits and vegetables with depression may be explained by: (i) recall and misclassification bias; (ii) inaccurate report of type, variety and amount of plant foods and (iii) quantitatively low intake of fruits and vegetables even in the highest tertiles of consumption. 
Furthermore, reverse causality common to cross-sectional studies could also be behind these contradictory results. Hence, future versions of the CNHS should include better design, methods, training and questionnaires for the dietary intake assessment.

Vitamins and minerals are nutrients found mainly in fruits and vegetables but also in foods like fish and whole grains. Some studies had associated elevated homocysteine levels and depressive symptoms ${ }^{(50,51)}$. Particularly, homocysteine is directly linked with vitamin $\mathrm{B}_{12}$ and folic acid. Elevated plasma homocysteine levels are common in older adults. A meta-analysis showed that older adults with higher homocysteine concentration had a higher risk of depression ${ }^{(51)}$. However, supplementation studies with vitamin $\mathrm{B}_{12}$ and folic acid on depression are heterogeneous, scarce and inconclusive ${ }^{(52)}$. Until now, multivita$\mathrm{min} /$ mineral supplementation as prevention or therapy for depression is not recommended ${ }^{(53-55)}$. Further studies are needed in B-vitamins in relation to depression in older adults ${ }^{(56)}$. Last, a recent cross-sectional study showed that higher dietary vitamin $\mathrm{K}$ intake was associated with lower depressive symptoms, underscoring the need for future research ${ }^{(57)}$.

Overall, healthy dietary patterns were inversely associated with the risk for depression and particularly Mediterranean diet is one of the dietary patterns with most of the evidence $^{(58,59)}$. The Chilean Food-Based Dietary Guidelines were elaborated following key recommendations aligned with the Mediterranean diet pattern and dietary questionnaires of the Survey were designed accordingly ${ }^{(17)}$. The major difference and cultural adaptation between the Mediterranean diet and the healthy eating score is regarding dairy products. While in a Mediterranean diet, the consumption of dairy products is negatively scored, the National dietary guidelines consider dairy products as healthy. The differentiation between the types of dairy products is of major relevance as whole milk and fatty dairy products were associated with negative health outcomes, while skimmed milk and fermented dairy products may be considered as healthy. Thus, further studies in LatinAmerican populations could include what type of dairy products was consumed ${ }^{(60)}$. Nonetheless, dairy product consumption was not a significant contributor to the association between the healthy eating score and depression. Randomised controlled trial studies support a healthy diet such as the Mediterranean to prevent mental health disorders and improve cognitive function ${ }^{(61,62)}$, but further evidence is needed ${ }^{(53,61,63)}$.

Future studies should include a more accurate and comprehensive dietary assessment to determine the role of diet in depression development ${ }^{(12,64)}$. For example, high-fat diets are known to cause gut dysbiosis and induce metabolic endotoxemia. In contrast, some dietary components, such as fibres, vitamins and polyphenols, are beneficial for the gut ecosystem ${ }^{(65)}$. In addition, dysbiosis of the gut microbiota and increased gut permeability may contribute to depression ${ }^{(66)}$. In this context, the effect of a polyphenolrich dietary pattern on gut permeability and depressive symptoms could be expected ${ }^{(67,68)}$.

The present study followed a cross-sectional design, and from its design, we cannot infer causality. However, it is a National representative sample and is one of the first studies of its kind at a national scale in a South American population. Our analysis of depression was based on the CIDI-SF, which is a screening instrument to detect MDE and is not a diagnostic tool. However, it was previously validated, and its use is well spread across national surveys due to its easy implementation. It would be important to take into consideration which tools are used for studying the link between depression and diet. In this regard, the development of biomarkers of intake would be needed to better assess the association between diet and health outcomes, particularly in older adults, who may have difficulties filling dietary questionnaires. Another limitation comes from the fact that the dietary assessment from the CNHS did not allow us to include other food items in the healthy eating score, such as intake of pastries, processed foods, red meat, olive oil or nuts. An important limitation is also a challenge because older adults present several comorbidities that, on one hand, can accentuate depression, and, on the other hand may limit the adherence to a healthy dietary pattern. Therefore, a comprehensive assessment and nutritional approach are essential for future studies. Among the strengths of the present study are its National coverage and the use of a healthy dietary score, instead of single food items.

In conclusion, higher levels of a healthy diet score were associated with reduced odds for MDE in a national representative sample of older adults from Chile. The implementation of public health actions aiming to improve the dietary habits of the older adult population, emphasising higher intakes of legumes and seafood, may contribute to tackling the increasing prevalence of depression.

\section{Acknowledgements}

Acknowledgements: We thank all participants for their cooperation and the Chilean Health Ministry, the Sub secretary of Public Health and the Pontificia Universidad Católica de Chile for designing and conducting the third National Health Survey (2016-2017). All the results in the study are responsibility of the authors and the Chilean Health Ministry was not involved in the study. Financial Support: F. Lanuza receives financial support from the Chilean Government for doing his PhD by the National Agency for Research and Development (ANID)/ Food and Nutrition Doctoral Program/ DOCTORADO BECAS CHILE/2019 - 72200061. T. Meroño would like to thank the ayuda IJCI-2017-32534 financiada por el Ministerio de Ciencia e Innovación/Agencia Estatal de Investigación/ 10.13039/501100011033 (Spain). R. Zamora-Ros would like 
to thank the 'Miguel Servet' program (CPII20/00009) from the Institute of Health Carlos III (Spain) and the European Social Fund (ESF), and IDIBELL acknowledges support from the Generalists de Catalunya through the CERCA Program. Conflict of interest: The authors declare no conflicts of interest. Authorship: F.L.: conceptualisation, methodology, data curation and formal analysis, writing-original draft preparation, writing-reviewing and editing. T.M.: conceptualisation, supervision, writing-reviewing and editing. R.P.-R., C.C.-M., Y.C.-C., G.N., C.T.-P., N.L.-L., M.A.M.-S., S.S.-P., R.Z.-R., C.L.-A.: reviewing, commenting and editing. Ethics of human subject participation: This study was conducted according to the guidelines laid down in the Declaration of Helsinki, and all procedures involving research study participants were approved by the Ethics Committee of the Pontifical Universidad Católica de Chile (16-019). All participants signed the written consent form.

\section{Supplementary material}

For supplementary material accompanying this paper visit https://doi.org/10.1017/S1368980021004869

\section{References}

1. Kanasi E, Ayilavarapu S \& Jones J (2016) The aging population: demographics and the biology of aging. Periodontol $\mathbf{7 2}$, $13-18$.

2. World Health Organization (2018) Ageing and Health. https://www.who.int/news-room/fact-sheets/detail/ageingand-health (accessed January 2021).

3. James SL, Abate D, Abate KH et al. (2018) Global, regional, and national incidence, prevalence, and years lived with disability for 354 diseases and injuries for 195 countries and territories, 1990-2017: a systematic analysis for the global burden of disease study 2017. Lancet 392, $1789-1858$

4. Abbafati C, Abbas KM, Abbasi-Kangevari M et al. (2020) Global burden of 369 diseases and injuries in 204 countries and territories, 1990-2019: a systematic analysis for the global burden of disease study 2019. Lancet 396, 1204-1222.

5. Singh-Manoux A, Dugravot A, Fournier A et al. (2017) Trajectories of depressive symptoms before diagnosis of dementia: a 28-year follow-up study. JAMA Psychiatry $\mathbf{7 4}$, 712-718.

6. Markkula N, Zitko P, Peña S et al. (2017) Prevalence, trends, correlates and treatment of depression in Chile in 2003 to 2010. Soc Psychiatry Psychiatr Epidemiol 52, 399-409.

7. Nazar G, Ulloa N, Martínez-Sanguinetti MA et al. (2020) Diagnóstico médico de depresión se asocia a sospecha de deterioro cognitivo en adultos mayores (Medical diagnosis of depression is associated with cognitive impairment in older adults). Rev Med Chil 148, 947-955.

8. Bromet E, Andrade LH, Hwang I et al. (2011) Cross-national epidemiology of DSM-IV major depressive episode. BMC Med 9, 90.

9. Arias-de la Torre J, Vilagut G, Ronaldson A et al. (2021) Prevalence and variability of current depressive disorder in 27 European countries: a population-based study. Lancet Public Health 2667, 1-10.

10. Lassale C, Batty GD, Baghdadli A et al. (2019) Healthy dietary indices and risk of depressive outcomes: a systematic review and meta-analysis of observational studies. Mol Psychiatry 24, 965-986.

11. Limongi F, Siviero P, Bozanic A et al. (2020) The effect of adherence to the Mediterranean diet on late-life cognitive disorders: a systematic review. J Am Med Dir Assoc 21, 1402-1409.

12. Wu P-Y, Chen K-M \& Belcastro F (2020) Dietary patterns and depression risk in older adults: systematic review and metaanalysis. Nutr Rev 79, 976-987.

13. Elstgeest LEM, Winkens LHH, Penninx BWJH et al. (2019) Associations of depressive symptoms and history with three a priori diet quality indices in middle-aged and older adults. $J$ Affect Disord 249, 394-403.

14. Meller F, Meller L \& Schäfer A (2021) The influence of diet quality on depression among adults and elderly: a population-based study. I Affect Disord 282, 1076-1081.

15. Salazar-Fernández C, Palet D, Haeger PA et al. (2021) Covid-19 perceived impact and psychological variables as predictors of unhealthy food and alcohol consumption trajectories: the role of gender and living with children as moderators. Int J Environ Res Public Health 18, 4542.

16. Tapsell LC, Neale EP, Satija A et al. (2016) Foods, nutrients, and dietary patterns: interconnections and implications for dietary guidelines. Adv Nutr 7, 445-454.

17. Ministerio de Salud \& Gobierno de Chile (Health Ministery and Government of Chile) (2018) Encuesta Nacional de Salud 2016-2017 (National Health Survey 2016-2017). http://epi.minsal.cl/encuesta-nacional-de-salud-2015-2016/ (accessed June 2021).

18. Vicente B, Rioseco P, Saldivia S et al. (2002) Prevalence of psychiatric disorders in Chile. Rev Med Chil 130, 527-536.

19. Vicente B, Kohn R, Rioseco P et al. (2006) Lifetime and 12month prevalence of DSM-III-R disorders in the Chile psychiatric prevalence study. Am J Psychiatry 163, 1362-1370.

20. Ministerio de Salud \& Gobierno de Chile (Health Ministery and Government of Chile) (2010) Encuesta Nacional de Salud 2009-2010 (National Health Survey 2009-2010). http:// epi.minsal.cl/resultados-encuestas/ (accessed June 2021).

21. Kessler RC \& Ustün TB (2008) The World Health Organization composite international diagnostic interview. World Ment Health Surv Glob Perspect Epidemiol Ment Disord 7, 58-90.

22. Cortés SO, Hasbún IZ, González CGG et al. (2015) Diseño y validación de la imagen para la difusión e implementación de las guías alimentarias para la población chilena (Design and validation of the image for the diffusion and implementation of Chilean food-based dietary guidelines). Nutr Hosp 32, 582-589.

23. Koch M, Fitzpatrick AL, Rapp SR et al. (2019) Alcohol consumption and risk of dementia and cognitive decline among older adults with or without mild cognitive impairment. JAMA Netw Open 2, 1-13.

24. Concha-Cisternas Y, Lanuza F, Waddell H et al. (2019) Association between adiposity levels and cognitive impairment in the Chilean older adult population. J Nutr Sci $\mathbf{8}$, e33.

25. Bonaccio M, Di Castelnuovo A, Costanzo S et al. (2016) Adherence to the traditional Mediterranean diet and mortality in subjects with diabetes. Prospective results from the MOLISANI study. Eur J Prev Cardiol 23, 400-407.

26. Ahmad S, Moorthy MV, Demler OV et al. (2018) Assessment of risk factors and biomarkers associated with risk of cardiovascular disease among women consuming a Mediterranean diet. JAMA Netw Open 1, e185708.

27. Estrella ML, Durazo-Arvizu RA, Mattei J et al. (2020) Alternate healthy eating index is positively associated with cognitive function among middle-aged and older Hispanics/Latinos in the HCHS/SOL. J Nutr 150, 1478-1487.

28. Ministerio de Salud \& Gobierno de Chile (Health Ministery and Government of Chile) (2010) Encuesta Nacional de Consumo Alimentario en Chile (ENCA) (Chile National 
Food Consumption Survey). https://www.minsal.cl/enca/ (accessed May 2020).

29. Lutz M, Petzold G \& Albala C (2019) Considerations for the development of innovative foods to improve nutrition in older adults. Nutrients 11, 1-11.

30. Agüero SD \& Leiva AV (2015) Caracterización antropométrica, calidad y estilos de vida del anciano chileno octogenario (Study of the anthropometric characteristics, quality of life and lifestyles of Chilean octogenarians). Nutr Hosp 31, 2554-2560.

31. Mujica-Coopman MF, Navarro-Rosenblatt D, López-Arana S et al. (2020) Nutrition status in adult Chilean population: economic, ethnic and sex inequalities in a post-transitional country. Public Health Nutr 23, S39-S50.

32. de Albuquerque L \& Albala C (2021) The role of social integration in the consumption of fruit and vegetables among the elderly. Cienc Saude Colet 26, 3895-3906.

33. Comai S, Bertazzo A, Bailoni L et al. (2007) Protein and nonprotein (free and protein-bound) tryptophan in legume seeds. Food Chem 103, 657-661.

34. Waclawiková B \& El Aidy S (2018) Role of microbiota and tryptophan metabolites in the remote effect of intestinal inflammation on brain and depression. Pharmaceuticals 11, 63.

35. Qi Q, Li J, Yu B et al. (2021) Host and gut microbial tryptophan metabolism and type 2 diabetes: an integrative analysis of host genetics, diet, gut microbiome and circulating metabolites in cohort studies. Gut. Published Online First: 14 June. doi: 10.1136/gutjnl-2021-324053.

36. Gibson EL (2018) Tryptophan supplementation and serotonin function: genetic variations in behavioural effects. Proc Nutr Soc 77, 174-188.

37. Dehhaghi M, Kazemi Shariat Panahi H \& Guillemin GJ (2019) Microorganisms, tryptophan metabolism, and kynurenine pathway: a complex interconnected loop influencing human health status. Int J Tryptophan Res 12, 1178646919852996.

38. Swardfager W, Herrmann N, Mazereeuw G et al. (2013) Zinc in depression: a meta-analysis. Biol Psychiatry 74, 872-878.

39. Vashum KP, McEvoy M, Milton AH et al. (2014) Dietary zinc is associated with a lower incidence of depression: findings from two Australian cohorts. J Affect Disord 166, 249-257.

40. Doning A \& Hautzinger M (2021) Zinc as an adjunct to antidepressant medication: a meta-analysis with subgroup analysis for different levels of treatment response to antidepressants. Nutr Neurosci. Online Ahead of Print: 28 February. doi: 10.1080/1028415X.2021.1888205.

41. Da Silva LEM, De Santana MLP, Costa PRDF et al. (2021) Zinc supplementation combined with antidepressant drugs for treatment of patients with depression: a systematic review and meta-analysis. Nutr Rev 79, 1-12.

42. Berger M, Taylor S, Harriss L et al. (2020) Cross-sectional association of seafood consumption, polyunsaturated fatty acids and depressive symptoms in two Torres Strait communities. Nutr Neurosci 23, 353-362.

43. Sánchez-Villegas A, Álvarez-Pérez J, Toledo E et al. (2018) Seafood consumption, $n-3$ fatty acids intake, and life-time prevalence of depression in the PREDIMED-plus trial. Nutrients 10, 2000.

44. Bae JH \& Kim G (2018) Systematic review and meta-analysis of $n$-3-fatty acids in elderly patients with depression. Nutr Res 50, 1-9.

45. Parletta N, Zarnowiecki D, Cho J et al. (2019) A Mediterranean-style dietary intervention supplemented with fish oil improves diet quality and mental health in people with depression: a randomized controlled trial (HELFIMED). Nutr Neurosci 22, 474-487.

46. Sharifan P, Hosseini MS \& Sharifan A (2017) The interventional relationship between frequent fish consumption and depression symptoms in aging adults: a randomized controlled trial. Int J Geriatr Psychiatry 32, e116-e122.

47. Saghafian F, Malmir H, Saneei P et al. (2018) Fruit and vegetable consumption and risk of depression: accumulative evidence from an updated systematic review and meta-analysis of epidemiological studies. Br J Nutr 119, $1087-1101$.

48. Szabo de Edelenyi F, Philippe C, Druesne-Pecollo N et al. (2021) Depressive symptoms, fruit and vegetables consumption and urinary 3-indoxylsulfate concentration: a nested case-control study in the French Nutrinet-Sante cohort. Eur J Nutr 60, 1059-1069.

49. Grases G, Colom MA, Sanchis P et al. (2019) Possible relation between consumption of different food groups and depression. BMC Psychol 7, 1-6.

50. Castro F, Melgarejo J, Chavez CA et al. (2021) Total plasma homocysteine and depressive symptoms in older Hispanics. J Alzheimer's Dis 82, S263-S269.

51. Almeida OP, McCaul K, Hankey GJ et al. (2008) Homocysteine and depression in later life. Arch Gen Psychiatry 65, 12861294.

52. de Koning EJ, van der Zwaluw NL, van Wijngaarden JP et al. (2016) Effects of two-year vitamin $B_{12}$ and folic acid supplementation on depressive symptoms and quality of life in older adults with elevated homocysteine concentrations: additional results from the B-proof study, an RCT. Nutrients 8, 748 .

53. Bot M, Brouwer IA, Roca M et al. (2019) Effect of multinutrient supplementation and food-related behavioral activation therapy on prevention of major depressive disorder among overweight or obese adults with subsyndromal depressive symptoms: the MooDFOOD randomized clinical trial. JAMA 321, 858-868.

54. Li G, Mbuagbaw L, Samaan Z et al. (2014) Efficacy of vitamin D supplementation in depression in adults: a systematic review. J Clin Endocrinol Metab 99, 757-767.

55. Young LM, Pipingas A, White DJ et al. (2019) A systematic review and meta-analysis of B vitamin supplementation on depressive symptoms, anxiety, and stress: effects on healthy and 'at-risk' individuals. Nutrients 11, 2232.

56. Moore K, Hughes CF, Hoey L et al. (2019) B-vitamins in relation to depression in older adults over 60 years of age: the trinity ulster department of agriculture (TUDA) cohort study. J Am Med Dir Assoc 20, 551.e1-557.e1.

57. Bolzetta F, Veronese N, Stubbs B et al. (2019) The relationship between dietary vitamin $\mathrm{k}$ and depressive symptoms in late adulthood: a cross-sectional analysis from a large cohort study. Nutrients 11, 1-10.

58. Li Y, Lv MR, Wei YJ et al. (2017) Dietary patterns and depression risk: a meta-analysis. Psychiatry Res 253, 373-382.

59. Shafiei F, Salari-Moghaddam A, Larijani B et al. (2019) Adherence to the Mediterranean diet and risk of depression: a systematic review and updated meta-analysis of observational studies. Nutr Rev 77, 230-239.

60. Hernández Ruiz A, García-Villanova B, Guerra Hernández EJ et al. (2015) Description of indexes based on the adherence to the Mediterranean dietary pattern: a review. Nutr Hosp 32, 1872-1884.

61. Opie RS, O'Neil A, Itsiopoulos C et al. (2015) The impact of whole-of-diet interventions on depression and anxiety: a systematic review of randomised controlled trials. Public Health Nutr 18, 2074-2093.

62. Martínez-Lapiscina EH, Clavero P, Toledo E et al. (2013) Mediterranean diet improves cognition: the PREDIMEDNAVARRA randomised trial. J Neurol Neurosurg Psychiatry 84, 1318-1325.

63. Wu PY, Lin MY \& Tsai PS (2020) Alternate healthy eating index and risk of depression: a meta-analysis and systematic review. Nutr Neurosci 23, 101-109. 
64. Jayedi A, Soltani S, Abdolshahi A et al. (2020) Healthy and unhealthy dietary patterns and the risk of chronic disease: an umbrella review of meta-analyses of prospective cohort studies. Br J Nutr 124, 1133-1144.

65. Fuke N, Nagata N, Suganuma H et al. (2019) Regulation of gut microbiota and metabolic endotoxemia with dietary factors. Nutrients 11, 2277.

66. Klimova B, Novotny M \& Valis M (2020) The impact of nutrition and intestinal microbiome on elderly depression - a systematic review. Nutrients 12, 710 .
67. Kontogianni MD, Vijayakumar A, Rooney C et al. (2020) A high polyphenol diet improves psychological well-being: the polyphenol intervention trial (pphit). Nutrients 12, $1-16$.

68. Peron G, Hidalgo Liberona N, González-Domínguez R et al. (2020) Exploring the molecular pathways behind the effects of nutrients and dietary polyphenols on gut microbiota and intestinal permeability: a perspective on the potential of metabolomics and future clinical applications. J Agric Food Chem 68, 1780-1789. 